\title{
Geometric characterizations of virtually free groups
}

\author{
Vítor Araújo \\ Universidade Federal da Bahia, Instituto de Matemática, \\ Av. Adhemar de Barros, $S / N$, Ondina, \\ 40170-110 Salvador-BA, Brazil \\ email: vitor.d.araujo@ufba.br \\ Pedro V. Silva \\ Universidade Federal da Bahia, Instituto de Matemática, Brazil \\ and Centro de Matemática, Faculdade de Ciências, Universidade do Porto, \\ R. Campo Alegre 687, 4169-007 Porto, Portugal \\ email: pvsilva@fc.up.pt
}

September 25, 2018

2010 Mathematics Subject Classification: 20F67, 51M10, 54E35

Keywords: hyperbolic metric spaces, hyperbolic groups, virtually free groups

\begin{abstract}
Four geometric conditions on a geodesic metric space, which are stronger variants of classical conditions characterizing hyperbolicity, are proved to be equivalent. In the particular case of the Cayley graph of a finitely generated group, it is shown that they characterize virtually free groups.
\end{abstract}

\section{Introduction}

Finitely generated virtually free groups constitute an important subclass of hyperbolic groups. A group $G$ is virtually free if it has a free subgroup $F$ of finite index. If $G$ is finitely generated, we may assume the same for $F$.

Virtually free groups constitute probably the class of groups which admits the widest variety of characterizations. Following Diekert and Weiss [5, 4, we enumerate a few to indulge all tastes.

A finitely generated group $G=\langle A\rangle$ is virtually free if and only if:

- $G$ is the fundamental group of a finite graph of finite groups [11] (see also [17, Theorem 7.3]);

- $G$ acts on a connected locally finite graph of finite treewidth, with finitely many orbits and finite node stabilizers [5];

- the Cayley graph $\Gamma_{A}(G)$ has finite treewidth [12]; 
- $\Gamma_{A}(G)$ can be $k$-triangulated [14];

- there exists some $\varepsilon \geq 0$ such that, for all coterminal geodesic $\xi$ and path $\xi^{\prime}$ in $\Gamma_{A}(G), \operatorname{Im}(\xi) \subseteq$ $D_{\varepsilon}\left(\operatorname{Im}\left(\xi^{\prime}\right)\right)[1]$

- there exist some finite generating set $B$ of $G$ and some $k \geq 0$ such that every $k$-locally geodesic in $\Gamma_{B}(G)$ is a geodesic [8];

- $G$ admits a finite presentation by some geodesic rewriting system [8];

- the language of all words on $A \cup A^{-1}$ representing the identity is context-free (Muller-Schupp's Theorem [13, 6]);

- $G$ is the universal group of a finite pregroup [16;

- the monadic second-order theory of $\Gamma_{A}(G)$ is decidable [12, 14];

- $G$ admits a Stallings section [18].

More details can be found in [1, 4.

The above list gives evidence of the interest which was devoted by different authors to the Cayley graph of a virtually free group. However, to our best knowledge, no explicit results were published on the classical geometric conditions used to define hyperbolicity [10, [7, Proposition 2.21].

Thus in Section 3 we establish equivalent geometric conditions for geodesic metric spaces which will allow us to characterize finitely generated virtually free groups when we consider their Cayley graphs in Section 4.

Our starting point were three of the most common characterizations of hyperbolicity, using respectively thin geodesic triangles (Rips condition), the Gromov product (the original definition by Gromov) and the mesh of a geodesic triangle. We succeed on replacing thin geodesic triangles by thin geodesic polygons, and the mesh of a geodesic triangle by the mesh of an arbitrary triangle. For the Gromov product, we consider inequalities with arbitrary long sequences of group elements.

\section{Preliminaries}

We present in this section well-known facts regarding hyperbolic spaces and hyperbolic groups. The reader is referred to [3, 7] for details.

If $(X, d)$ is a metric space and $\varepsilon \geq 0$, we denote by $D_{\varepsilon}(x)$ the closed ball of center $x \in X$ and radius $\varepsilon$. If $Y \subseteq X$ is nonempty, we write

$$
D_{\varepsilon}(Y)=\bigcup_{y \in Y} D_{\varepsilon}(y) .
$$

A mapping $\varphi:(X, d) \rightarrow\left(X^{\prime}, d^{\prime}\right)$ between metric spaces is called an isometric embedding if $d^{\prime}(x \varphi, y \varphi)=d(x, y)$ for all $x, y \in X$. A surjective isometric embedding is an isometry.

We consider the usual metric for $\mathbb{R}$ and its subsets. A path in $(X, d)$ is a continuous mapping $\xi:[0, s] \rightarrow X\left(s \in \mathbb{R}_{0}^{+}\right)$. If $\xi^{\prime}:\left[0, s^{\prime}\right] \rightarrow X$ is another path and $0 \xi^{\prime}=0 \xi, s^{\prime} \xi^{\prime}=s \xi$, we say that the paths $\xi$ and $\xi^{\prime}$ are coterminal.

A metric space $(X, d)$ is said to be geodesic if, for all $x, y \in X$, there exists some path $\xi:[0, s] \rightarrow X$ which is an isometric embedding and such that $0 \xi=x$ and $s \xi=y$. We call $\xi$ a geodesic of $(X, d)$. 
A quasi-isometric embedding of metric spaces is a mapping $\varphi:(X, d) \rightarrow\left(X^{\prime}, d^{\prime}\right)$ such that there exist constants $\lambda \geq 1$ and $K \geq 0$ satisfying

$$
\frac{1}{\lambda} d(x, y)-K \leq d^{\prime}(x \varphi, y \varphi) \leq \lambda d(x, y)+K
$$

for all $x, y \in X$. We may call it a $(\lambda, K)$-quasi-isometric embedding if we want to stress the constants. If in addition

$$
\forall x^{\prime} \in X^{\prime} \exists x \in X: d^{\prime}\left(x^{\prime}, x \varphi\right) \leq K,
$$

we say that $\varphi$ is a quasi-isometry.

Two metric spaces $(X, d)$ and $\left(X^{\prime}, d^{\prime}\right)$ are said to be quasi-isometric if there exists a quasi-isometry $\varphi:(X, d) \rightarrow\left(X^{\prime}, d^{\prime}\right)$. Quasi-isometry turns out to be an equivalence relation on the class of metric spaces. A path $\xi:[0, s] \rightarrow X$ which is a quasi-isometric embedding is called a quasi-geodesic of $(X, d)$.

Let $(X, d)$ be a geodesic metric space. A triangle in $(X, d)$ is a collection $\Delta=\left[\left[\xi_{0}, \xi_{1}, \xi_{2}\right]\right]$ of three paths $\xi_{i}:\left[0, s_{i}\right] \rightarrow X$ such that

$$
s_{i} \xi_{i}=0 \xi_{i+1} \quad \text { for } i=0,1,2 .
$$

Here and in many other instances of the paper, we consider the indices modulo 3 (or $n+1$ ), so that $\xi_{3}=\xi_{0}$ in the formula above. If all the paths $\xi_{i}$ are geodesics, we say that $\Delta$ is a geodesic triangle.

More generally, we define a geodesic polygon to be a collection of $n+1$ geodesics $\Pi=\left[\left[\xi_{0}, \xi_{1}, \ldots, \xi_{n}\right]\right]$ such that

$$
s_{i} \xi_{i}=0 \xi_{i+1} \quad \text { for } i=0,1, n,
$$

with $n \geq 1$ arbitrary. We may call $\Pi$ an $(n+1)$-gon. If $n=1$, we have a geodesic bigon.

Given $\delta \geq 0$, a geodesic polygon $\left[\left[\xi_{0}, \xi_{1}, \ldots, \xi_{n}\right]\right]$ is said to be $\delta$-thin if

$$
\operatorname{Im}\left(\xi_{n}\right) \subseteq D_{\delta}\left(\operatorname{Im}\left(\xi_{0}\right) \cup \operatorname{Im}\left(\xi_{n-1}\right)\right) .
$$

We say that $(X, d)$ is $\delta$-hyperbolic if every geodesic triangle in $(X, d)$ is $\delta$-thin. We say that $(X, d)$ is hyperbolic if $(X, d)$ is $\delta$-hyperbolic for some $\delta \geq 0$.

There are several equivalent characterizations of hyperbolicity. The original one, introduced by Gromov in [10], uses the concept of Gromov product, which we now define. We note that it can be defined for every metric space $(X, d)$.

Given $x, y, p \in X$, we define

$$
(x \mid y)_{p}=\frac{1}{2}(d(p, x)+d(p, y)-d(x, y)) .
$$

We say that $(x \mid y)_{p}$ is the Gromov product of $x$ and $y$, taking $p$ as basepoint.

A third road to hyperbolicity uses the concept of mesh. Recall the notion of diameter. Given a nonempty $Y \subseteq X$, we write

$$
\operatorname{diam}(Y)=\sup \left\{d\left(y, y^{\prime}\right) \mid y, y^{\prime} \in Y\right\} .
$$

Given a triangle $\Delta=\left[\left[\xi_{0}, \xi_{1}, \xi_{2}\right]\right]$ in a geodesic metric space $(X, d)$, we define

$$
\operatorname{mesh}(\Delta)=\inf \left\{\operatorname{diam}\left(\left\{u_{0}, u_{1}, u_{2}\right\}\right) \mid u_{i} \in \operatorname{Im}\left(\xi_{i}\right)(i=0,1,2)\right\}
$$

The following equivalences are well known, see [7, Proposition 2.21] for a proof. 
Theorem 2.1 The following conditions are equivalent for a geodesic metric space $(X, d)$ :

(i) $(X, d)$ is hyperbolic;

(ii) there exists some $\delta^{\prime} \geq 0$ such that

$$
\left(x_{0} \mid x_{2}\right)_{p} \geq \min \left\{\left(x_{0} \mid x_{1}\right)_{p},\left(x_{1} \mid x_{2}\right)_{p}\right\}-\delta^{\prime}
$$

for all $x_{0}, x_{1}, x_{2}, p \in X ;$

(iii) there exists some $\mu \geq 0$ such that

$$
\operatorname{mesh}(\Delta) \leq \mu
$$

for every geodesic triangle $\Delta$ in $(X, d)$.

Among the most important properties of hyperbolic spaces, stands the fact that they are closed under quasi-isometry.

Given $Y, Z \subseteq X$ nonempty, the Hausdorff distance between $Y$ and $Z$ is defined by

$$
\operatorname{Haus}(Y, Z)=\max \left\{\sup _{y \in Y} d(y, Z), \sup _{z \in Z} d(z, Y)\right\} \text {. }
$$

The following result of Gromov [10] (see also [7, Theorem 5.4.21]) is also important to us:

Proposition 2.2 Let $\lambda \geq 1$ and $K, \delta \geq 0$. There exists a constant $R(\delta, \lambda, K) \geq 0$ such that: if $(X, d)$ is a $\delta$-hyperbolic space, $\xi$ is a geodesic and $\xi^{\prime}$ a coterminal $(\lambda, K)$-quasi-geodesic in $(X, d)$, then

$$
\operatorname{Haus}\left(\operatorname{Im}(\xi), \operatorname{Im}\left(\xi^{\prime}\right)\right) \leq R(\delta, \lambda, K) .
$$

We proceed now to define hyperbolic groups.

Given a subset $A$ of a group $G$, we denote by $\langle A\rangle$ the subgroup of $G$ generated by $A$. We assume throughout the paper that generating sets are finite.

Given $G=\langle A\rangle$, we write $\widetilde{A}=A \cup A^{-1}$. The Cayley graph $\Gamma_{A}(G)$ has vertex set $G$ and edges of the form $g \stackrel{a}{\longrightarrow} g a$ for all $g \in G$ and $a \in \widetilde{A}$. The geodesic metric $d_{A}$ on $G$ is defined by taking $d_{A}(g, h)$ to be the length of the shortest path connecting $g$ to $h$ in $\Gamma_{A}(G)$.

Since $\operatorname{Im}\left(d_{A}\right) \subseteq \mathbb{N}$, then $\left(G, d_{A}\right)$ is not a geodesic metric space. However, we can remedy that by embedding $\left(G, d_{A}\right)$ isometrically into the geometric realization $\bar{\Gamma}_{A}(G)$ of $\Gamma_{A}(G)$, when vertices become points and edges become segments of length 1 in some (euclidean) space, intersections being determined by adjacency only. With the obvious metric, $\bar{\Gamma}_{A}(G)$ is a geodesic metric space, and the geometric realization is unique up to isometry. We denote also by $d_{A}$ the induced metric on $\bar{\Gamma}_{A}(G)$.

We say that the group $G$ is hyperbolic if the geodesic metric space $\left(\bar{\Gamma}_{A}(G), d_{A}\right)$ is hyperbolic. Since $\left(\bar{\Gamma}_{A}(G), d_{A}\right)$ is quasi-isometric to $\left(\bar{\Gamma}_{B}(G), d_{B}\right)$ for every alternative finite generating set $B$ of $G$, the concept of hyperbolic group is independent from the finite generating set considered. However, the hyperbolicity constant $\delta$ may vary with the generating set.

Let $H$ be a subgroup of a hyperbolic group $G=\langle A\rangle$ and let $q \geq 0$. We say that $H$ is $q$-quasiconvex with respect to $A$ if, for every geodesic $\xi:[0, s] \rightarrow \bar{\Gamma}_{A}(G)$ with $0 \xi, s \xi \in H$, we have

$$
\operatorname{Im}(\xi) \subseteq D_{q}(H)
$$

We say that $H$ is quasi-convex if it is $q$-quasi-convex for some $q \geq 0$. Like most other properties in the theory of hyperbolic groups, quasi-convex does not depend on the finite generating set considered.

A (finitely generated) subgroup of a hyperbolic group needs not be hyperbolic, but a quasi-convex subgroup of a hyperbolic group is always hyperbolic. The converse is not true in general. The reader is referred to [3, Section III.Г.3] for details on quasi-convex subgroups. 


\section{$3 \quad$ Geodesic metric spaces}

We prove in this section the equivalence of four geometric conditions on a geodesic metric space.

Given $\delta \geq 0$, we say that a geodesic metric space $(X, d)$ is polygon $\delta$-hyperbolic if every geodesic polygon in $(X, d)$ is $\delta$-thin. We say that $(X, d)$ is polygon hyperbolic if it is polygon $\delta$-hyperbolic for some $\delta \geq 0$. We introduce also the notation

$$
\operatorname{mesh}(X, d)=\sup \{\operatorname{mesh}(\Delta) \mid \Delta \text { is a triangle in }(X, d)\} .
$$

Lemma 3.1 Let $\varphi:(X, d) \rightarrow\left(X^{\prime}, d^{\prime}\right)$ be a quasi-isometric embedding of geodesic metric spaces. Then:

(i) if $\left(X^{\prime}, d^{\prime}\right)$ is polygon hyperbolic, so is $(X, d)$;

(ii) if $\operatorname{mesh}\left(X^{\prime}, d^{\prime}\right)$ is finite, so is $\operatorname{mesh}(X, d)$.

Proof. (i) Assume that $\varphi$ is a $(\lambda, K)$-quasi-isometry and $\left(X^{\prime}, d^{\prime}\right)$ is polygon $\delta^{\prime}$-hyperbolic. Let $\left[\left[\xi_{0}, \ldots, \xi_{n}\right]\right]$ be a geodesic polygon in $(X, d)$.

Each geodesic $\xi_{i}$ induces a $(\lambda, K)$-quasi-geodesic $\xi_{i} \varphi$ in $X^{\prime}$. For $i=0, \ldots, n$, let $\xi_{i}^{\prime}$ be a geodesic in $X^{\prime}$ coterminal with $\xi_{i} \varphi$. Since $\left(X^{\prime}, d^{\prime}\right)$ is $\delta^{\prime}$-hyperbolic, it follows from Proposition [2.2 that

$$
\operatorname{Haus}\left(\operatorname{Im}\left(\xi_{i} \varphi\right), \operatorname{Im}\left(\xi_{i}^{\prime}\right)\right) \leq R(\delta, \lambda, K)
$$

for $i=0, \ldots, n$.

Write $R=R(\delta, \lambda, K)$ and let $y \in \operatorname{Im}\left(\xi_{n}\right)$. Then $y \varphi \in \operatorname{Im}\left(\xi_{n} \varphi\right)$ and by (11) we have $d^{\prime}\left(y \varphi, y^{\prime}\right) \leq R$ for some $y^{\prime} \in \operatorname{Im}\left(\xi_{n}^{\prime}\right)$.

Now $\left[\left[\xi_{0}^{\prime}, \ldots, \xi_{n}^{\prime}\right]\right]$ is a geodesic polygon in $X^{\prime}$. Since $\left(X^{\prime}, d^{\prime}\right)$ is polygon $\delta^{\prime}$-hyperbolic, there exist $j \in\{0, \ldots, n-1\}$ and $z^{\prime} \in \operatorname{Im}\left(\xi_{j}^{\prime}\right)$ such that $d^{\prime}\left(y^{\prime}, z^{\prime}\right) \leq \delta^{\prime}$. By (1), we have $d^{\prime}\left(z^{\prime}, z \varphi\right) \leq R$ for some $z \in \operatorname{Im}\left(\xi_{j}\right)$, hence

$$
\frac{1}{\lambda} d(y, z)-K \leq d^{\prime}(y \varphi, z \varphi)
$$

yields

$$
d(y, z) \leq \lambda\left(d^{\prime}(y \varphi, z \varphi)+K\right) \leq \lambda\left(d^{\prime}\left(y \varphi, y^{\prime}\right)+d^{\prime}\left(y^{\prime}, z^{\prime}\right)+d^{\prime}\left(z^{\prime}, z \varphi\right)+K\right) \leq \lambda\left(2 R+\delta^{\prime}+K\right) .
$$

Since $y \in \operatorname{Im}\left(\xi_{n}\right)$ is arbitrary and $z \in \operatorname{Im}\left(\xi_{j}\right)$, it follows that $(X, d)$ is polygon $\delta$-hyperbolic for $\delta=\lambda\left(2 R+\delta^{\prime}+K\right)$.

(ii) Assume that $\varphi$ is a $(\lambda, K)$-quasi-isometry and $\operatorname{mesh}\left(X^{\prime}, d^{\prime}\right)=\mu^{\prime}<+\infty$. Let $\left[\left[\xi_{0}, \xi_{1}, \xi_{2}\right]\right]$ be a triangle in $(X, d)$. Then $\left[\left[\xi_{0} \varphi, \xi_{1} \varphi, \xi_{2} \varphi\right]\right]$ is a triangle in $\left(X^{\prime}, d^{\prime}\right)$. Since mesh $\left(X^{\prime}, d^{\prime}\right)=\mu^{\prime}$, there exist some $u_{i} \in \operatorname{dom}\left(\xi_{i} \varphi\right)=\operatorname{dom}\left(\xi_{i}\right)$, for $i=0,1,2$, such that

$$
\operatorname{diam}\left(\left\{u_{0} \xi_{0} \varphi, u_{1} \xi_{1} \varphi, u_{2} \xi_{2} \varphi\right\}\right) \leq \mu^{\prime} .
$$

Since

$$
\frac{1}{\lambda} d\left(u_{i} \xi_{i}, u_{j} \xi_{j}\right)-K \leq d^{\prime}\left(u_{i} \xi_{i} \varphi, u_{j} \xi_{j} \varphi\right)
$$

for all $i, j \in\{0,1,2\}$, we get

$$
\operatorname{diam}\left(\left\{u_{0} \xi_{0}, u_{1} \xi_{1}, u_{2} \xi_{2}\right\}\right) \leq \lambda\left(\mu^{\prime}+K\right) .
$$

Therefore

$$
\operatorname{mesh}(X, d) \leq \lambda\left(\mu^{\prime}+K\right)<+\infty
$$


Theorem 3.2 Let; $(X, d)$ be a geodesic metric space. Then the following conditions are equivalent:

(i) $(X, d)$ is polygon hyperbolic;

(ii) there exists some $\delta^{\prime} \geq 0$ such that

$$
\left(x_{0} \mid x_{n}\right)_{p} \geq \min \left\{\left(x_{0} \mid x_{1}\right)_{p}, \ldots\left(x_{n-1} \mid x_{n}\right)_{p}\right\}-\delta^{\prime}
$$

holds for all $n \geq 2$ and $x_{0}, \ldots, x_{n}, p \in X$;

(iii) there exists some $\varepsilon \geq 0$ such that, for all coterminal geodesic $\xi$ and path $\xi^{\prime}$ in $(X, d)$,

$$
\operatorname{Im}(\xi) \subseteq D_{\varepsilon}\left(\operatorname{Im}\left(\xi^{\prime}\right)\right)
$$

(iv) $\operatorname{mesh}(X, d)<+\infty$.

Proof. (i) $\Rightarrow$ (ii). Assume that $(X, d)$ is polygon $\delta$-hyperbolic. Let $x_{0}, \ldots, x_{n}, p \in X$ with $n \geq 2$. For $i=0, \ldots, n$, consider a geodesic $\xi_{i}:\left[0, s_{i}\right] \rightarrow X$ such that $0 \xi_{i}=x_{i}$ and $s_{i} \xi_{i}=x_{i+1}$. Let $\Pi=\left[\left[\xi_{0}, \ldots, \xi_{n}\right]\right]$ and $Z=\operatorname{Im}\left(\xi_{0}\right) \cup \ldots \cup \operatorname{Im}\left(\xi_{n-1}\right)$.

Since $(X, d)$ is polygon $\delta$-hyperbolic, it is in particular $\delta$-hyperbolic. By [19, Lemmas 2.9, 2.31 and 2.32], we have

$$
\left(x_{i} \mid x_{i+1}\right)_{p} \leq d\left(p, \operatorname{Im}\left(\xi_{i}\right)\right) \leq\left(x_{i} \mid x_{i+1}\right)_{p}+2 \delta
$$

for $i=0, \ldots, n$. Hence

$$
\min \left\{\left(x_{0} \mid x_{1}\right)_{p}, \ldots\left(x_{n-1} \mid x_{n}\right)_{p}\right\} \leq \min \left\{d\left(p, \operatorname{Im}\left(\xi_{0}\right)\right), \ldots, d\left(p, \operatorname{Im}\left(\xi_{n-1}\right)\right)\right\}=d(p, Z) .
$$

Since $\operatorname{Im}\left(\xi_{n}\right)$ is compact, we have $d\left(p, \operatorname{Im}\left(\xi_{n}\right)\right)=d(p, q)$ for some $q \in \operatorname{Im}\left(\xi_{n}\right)$. Applying our hypothesis to $\Pi$ we get $d(q, Z) \leq \delta$ and so

$$
d(p, Z) \leq d(p, q)+d(q, Z) \leq d\left(p, \operatorname{Im}\left(\xi_{n}\right)\right)+\delta \leq\left(x_{n} \mid x_{0}\right)_{p}+3 \delta
$$

in view of (2). Now (3) and (4) together yield

$$
\left(x_{0} \mid x_{n}\right)_{p} \geq d(p, Z)-3 \delta \geq \min \left\{\left(x_{0} \mid x_{1}\right)_{p}, \ldots\left(x_{n-1} \mid x_{n}\right)_{p}\right\}-3 \delta
$$

and we are done.

(ii) $\Rightarrow\left(\right.$ i). Let $\left[\left[\xi_{0}, \ldots, \xi_{n}\right]\right]$ be a geodesic polygon in $(X, d)$ with geodesics $\xi_{i}:\left[0, s_{i}\right] \rightarrow X$ for $i=0, \ldots, n$. Write $x_{i}=0 \xi_{i}=s_{i-1} \xi_{i-1}$ for $i=0, \ldots, n$. Let $p \in \operatorname{Im}\left(\xi_{n}\right)$ and let $Z=\operatorname{Im}\left(\xi_{0}\right) \cup \ldots \cup$ $\operatorname{Im}\left(\xi_{n-1}\right)$.

By [19, Lemmas 2.9, 2.30 and 2.32], the case $n=2$ in condition (ii) implies

$$
\left(x_{i} \mid x_{i+1}\right)_{p} \leq d\left(p, \operatorname{Im}\left(\xi_{i}\right)\right) \leq\left(x_{i} \mid x_{i+1}\right)_{p}+2 \delta^{\prime}
$$

for $i=0, \ldots, n$. Hence

$$
d(p, Z)=\min \left\{d\left(p, \operatorname{Im}\left(\xi_{0}\right)\right), \ldots, d\left(p, \operatorname{Im}\left(\xi_{n-1}\right)\right)\right\} \leq \min \left\{\left(x_{0} \mid x_{1}\right)_{p}, \ldots,\left(x_{n-1} \mid x_{n}\right)_{p}\right\}+2 \delta^{\prime}
$$

By condition (ii), we get

$$
d(p, Z) \leq\left(x_{0} \mid x_{n}\right)_{p}+3 \delta^{\prime} .
$$


Since $p \in \operatorname{Im}\left(\xi_{n}\right)$, it follows from (5) that $d(p, Z) \leq 3 \delta^{\prime}$. Therefore $(X, d)$ is polygon $3 \delta^{\prime}$-hyperbolic.

(i) $\Rightarrow$ (iii). Assume that $(X, d)$ is polygon $\delta$-hyperbolic. Let $\xi:[0, s] \rightarrow X$ be a geodesic and $\xi^{\prime}:\left[0, s^{\prime}\right] \rightarrow X$ be a coterminal path. Since $\operatorname{Im}\left(\xi^{\prime}\right)$ is compact, we can find finitely many $x_{0}=s \xi, x_{1}, \ldots, x_{n-1}, x_{n}=0 \xi \in \operatorname{Im}\left(\xi^{\prime}\right)$ such that $d\left(x_{i}, x_{i-1}\right) \leq 1$ for $i=1, \ldots, n$. Now we build a geodesic polygon $\Pi=\left[\left[\xi_{0}, \ldots, \xi_{n}\right]\right]$, where each $\xi_{i}$ is a geodesic with endpoints $x_{i}$ and $x_{i+1}$, and $\xi_{n}=\xi$.

Let $p \in \operatorname{Im}(\xi)$. Since $(X, d)$ is polygon $\delta$-hyperbolic, there exist some $j \in\{0, \ldots, n-1\}$ and $q \in \operatorname{Im}\left(\xi_{j}\right)$ such that $d(p, q) \leq \delta$. Hence

$$
d\left(p, \operatorname{Im}\left(\xi^{\prime}\right) \leq d\left(p, x_{j}\right) \leq d(p, q)+d\left(q, x_{j}\right) \leq \delta+1\right.
$$

and so $\operatorname{Im}(\xi) \subseteq D_{\delta+1}\left(\operatorname{Im}\left(\xi_{i}^{\prime}\right)\right)$.

(iii) $\Rightarrow$ (iv). If we consider a geodesic triangle $\left[\left[\xi_{0}, \xi_{1}, \xi_{2}\right]\right]$ in $(X, d)$ and view $\operatorname{Im}\left(\xi_{0}\right) \cup \operatorname{Im}\left(\xi_{1}\right)$ as the image of a single path, coterminal with $\xi_{2}$, it follows from condition (iii) that $(X, d)$ is $\varepsilon$-hyperbolic. Therefore, by Theorem 2.1, there exists some $\mu \geq 0$ such that

$$
\operatorname{mesh}(\Delta) \leq \mu
$$

for every geodesic triangle $\Delta$ in $(X, d)$.

Let $\Delta^{\prime}=\left[\left[\xi_{0}^{\prime}, \xi_{1}^{\prime}, \xi_{2}^{\prime}\right]\right]$ be a triangle in $(X, d)$. Consider a geodesic triangle $\Delta=\left[\left[\xi_{0}, \xi_{1}, \xi_{2}\right]\right]$ where $\xi_{i}$ and $\xi_{i}^{\prime}$ are coterminal for $i=0,1,2$. Since $\operatorname{mesh}(\Delta) \leq \mu$, there exist $p_{i} \in \operatorname{Im}\left(\xi_{i}\right)$ such that

$$
\left.\operatorname{diam}\left(\left\{p_{0}, p_{1}, p_{2}\right)\right\}\right) \leq \mu
$$

Now, also by condition (iii), there exist $q_{i} \in \operatorname{Im}\left(\xi_{i}^{\prime}\right)$ such that $d\left(p_{i}, q_{i}\right) \leq \varepsilon$ for $i=0,1,2$. Therefore, for all $i, j \in\{0,1,2\}$, we get

$$
d\left(q_{i}, q_{j}\right) \leq d\left(q_{i}, p_{i}\right)+d\left(p_{i}, p_{j}\right)+d\left(p_{j}, q_{j}\right) \leq 2 \varepsilon+\mu .
$$

Therefore $\operatorname{mesh}(X, d) \leq 2 \varepsilon+\mu$ and (iv) holds.

(iv) $\Rightarrow$ (i). Assume that $\operatorname{mesh}(X, d)=\mu<+\infty$. Let $\Pi=\left[\left[\xi_{0}, \ldots, \xi_{n}\right]\right]$ be a geodesic polygon in $(X, d)$ with geodesics $\xi_{i}:\left[0, s_{i}\right] \rightarrow X$ for $i=0, \ldots, n$. Write $x_{i}=0 \xi_{i}=s_{i-1} \xi_{i-1}$ for $i=0, \ldots, n$. Let $p \in \operatorname{Im}\left(\xi_{n}\right)$, say $p=y \xi_{n}$, and let $Z=\operatorname{Im}\left(\xi_{0}\right) \cup \ldots \cup \operatorname{Im}\left(\xi_{n-1}\right)$. We prove that $d(p, Z) \leq \frac{3 \mu+1}{2}$.

We may of course assume that $d\left(p, x_{0}\right), d\left(p, x_{n}\right) \geq \frac{\mu+1}{2}$. We can build three paths $\xi_{0}^{\prime}, \xi_{1}^{\prime}, \xi_{2}^{\prime}$ satisfying the following conditions:

- $\operatorname{Im}\left(\xi_{0}^{\prime}\right)=\left[y+\frac{\mu+1}{2}, s_{n}\right] \xi_{n} \cup Z$ and has endpoints $\left(y+\frac{\mu+1}{2}\right) \xi_{n}$ and $x_{n}$;

- $\operatorname{Im}\left(\xi_{1}^{\prime}\right)=\left[0, y-\frac{\mu+1}{2}\right] \xi_{n}$ and has endpoints $x_{n}$ and $\left(y-\frac{\mu+1}{2}\right) \xi_{n}$;

- $\operatorname{Im}\left(\xi_{2}^{\prime}\right)=\left[y-\frac{\mu+1}{2}, y+\frac{\mu+1}{2}\right] \xi_{n}$ and has endpoints $\left(y-\frac{\mu+1}{2}\right) \xi_{n}$ and $\left(y+\frac{\mu+1}{2}\right) \xi_{n}$.

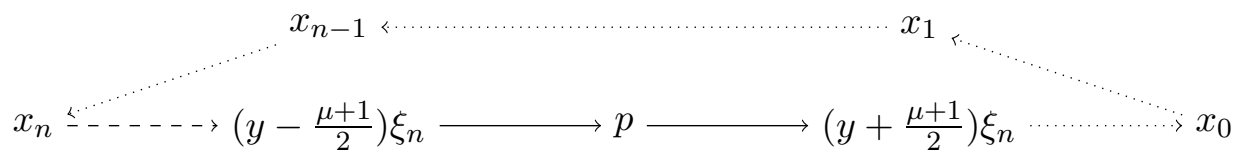


Let $\Delta=\left[\left[\xi_{0}^{\prime}, \xi_{1}^{\prime}, \xi_{2}^{\prime}\right]\right]$. Since mesh $(\Pi) \leq \mu$, there exist $u_{i} \in \operatorname{Im}\left(\xi_{i}^{\prime}\right)$ such that $\operatorname{diam}\left(\left\{u_{0}, u_{1}, u_{2}\right\}\right) \leq \mu$. Suppose that $u_{0} \notin Z$. Then $u_{0} \in\left[y+\frac{\mu+1}{2}, s_{n}\right] \xi_{n}$. Since $u_{1} \in\left[0, y-\frac{\mu+1}{2}\right] \xi_{n}$ and $\xi_{n}$ is a geodesic, this contradicts $d\left(u_{0}, u_{1}\right) \leq \mu$. Hence $u_{0} \in Z$ and we get

$$
d(p, Z) \leq d\left(p, u_{0}\right) \leq d\left(p, u_{2}\right)+d\left(u_{2}, u_{0}\right) \leq \frac{\mu+1}{2}+\mu=\frac{3 \mu+1}{2}
$$

as claimed. Therefore $(X, d)$ is polygon $\frac{\mu+1}{2}$-hyperbolic.

\section{Virtually free groups}

We present next several equivalent geometric characterizations of virtually free groups. We only have found out that the equivalence (i) $\Leftrightarrow$ (iv) had been previously proved by Antolin in [1] after we had written our own proof of the theorem, so we decided to keep our alternative proof of the equivalence (i) $\Leftrightarrow$ (ii).

We denote by $(g \mid h)_{p}^{A}$ the Gromov product of $g, h \in G$ with basepoint $p$ in $\left(\bar{\Gamma}_{A}(G), d_{A}\right)$.

Theorem 4.1 Let $G=\langle A\rangle$ be a finitely generated group. Then the following conditions are equivalent:

(i) $G$ is virtually free;

(ii) $\bar{\Gamma}_{A}(G)$ is polygon hyperbolic;

(iii) there exists some $\delta^{\prime} \geq 0$ such that

$$
\left(g_{0} \mid g_{n}\right)_{p}^{A} \geq \min \left\{\left(g_{0} \mid g_{1}\right)_{p}^{A}, \ldots\left(g_{n-1} \mid g_{n}\right)_{p}^{A}\right\}-\delta^{\prime}
$$

for all $n \geq 2$ and $g_{0}, \ldots, g_{n}, p \in G$;

(iv) there exists some $\varepsilon \geq 0$ such that, for all coterminal geodesic $\xi$ and path $\xi^{\prime}$ in $\left(\bar{\Gamma}_{A}(G), d_{A}\right)$,

$$
\operatorname{Im}(\xi) \subseteq D_{\varepsilon}\left(\operatorname{Im}\left(\xi^{\prime}\right)\right)
$$

(v) $\operatorname{mesh}\left(\bar{\Gamma}_{A}(G), d_{A}\right)<+\infty$.

Proof. (i) $\Rightarrow$ (ii). Since $G$ is virtually free and finitely generated, $G$ has a finite index free subgroup $F$ of finite rank. By [7, Proposition 1.11], there exists a finite generating set $B$ of $F$ such that $\bar{\Gamma}_{A}(G)$ is quasi-isometric to $\bar{\Gamma}_{B}(F)$. Let $C$ be a basis of $F$. As mentioned in Section 2 , $\bar{\Gamma}_{B}(F)$ is quasi-isometric to $\bar{\Gamma}_{C}(F)$. Since $\bar{\Gamma}_{C}(F)$ is a tree, it is trivially polygon 0 -hyperbolic. Therefore, by Lemma 3.1(i), $\bar{\Gamma}_{A}(G)$ is polygon hyperbolic.

(ii) $\Rightarrow$ (i). Since $\bar{\Gamma}_{A}(G)$ is polygon hyperbolic, it is in particular hyperbolic. Thus $G$ is a hyperbolic group. Suppose that $G$ is not virtually free. By a theorem of Bonk and Kleiner [2, Theorem 1], the hyperbolic plane $\mathbb{H}^{2}$ admits a quasi-isometric embedding into $\bar{\Gamma}_{A}(G)$. In view of Lemma 3.1(i), to reach the required contradiction it suffices to show that $\mathbb{H}^{2}$ is not polygon hyperbolic.

We shall use the Poincaré disk model of $\mathbb{H}^{2}$ (see [9, p.232] for details). We can view $\mathbb{H}^{2}$ as the open disk $\{z \in \mathbb{C}|| z \mid<1\}$. Geodesic lines are either diameters of the disk or arcs of a circle whose ends are perpendicular to the disk boundary. We denote by $d$ the Poincaré metric on $\mathbb{H}^{2}$.

Let $\varepsilon>0$. Using a diameter and a sufficiently large number of small circles, 


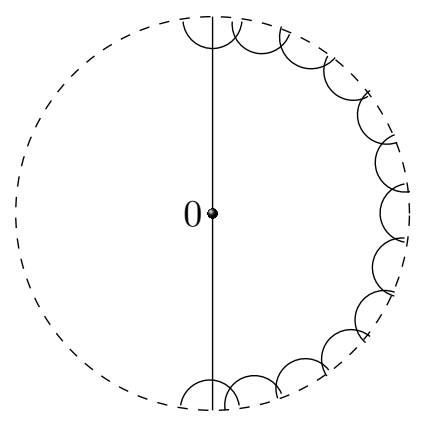

we can build a geodesic polygon $\left[\left[\xi_{0}, \ldots, \xi_{n}\right]\right]$ such that $\operatorname{Im}\left(\xi_{n}\right)$ is contained in the diameter and $|z|>1-\varepsilon$ for every $z \in \operatorname{Im}\left(\xi_{0}\right) \cup \ldots \cup \operatorname{Im}\left(\xi_{n-1}\right)$. Consider $0 \in \operatorname{Im}\left(\xi_{n}\right)$. Then

$$
d(0, z)=\left|\ln \frac{1-|z|}{1+|z|}\right|
$$

(see [9, p.248]). Since the euclidean distance from 0 to $\operatorname{Im}\left(\xi_{0}\right) \cup \ldots \cup \operatorname{Im}\left(\xi_{n-1}\right)$ tends to 1 when $\varepsilon$ tends to 0 , it follows that $d\left(0, \operatorname{Im}\left(\xi_{0}\right) \cup \ldots \cup \operatorname{Im}\left(\xi_{n-1}\right)\right)$ tends to $+\infty$ when $\varepsilon$ tends to 0 . Therefore $\mathbb{H}^{2}$ is not polygon hyperbolic.

(ii) $\Rightarrow$ (iii) and (ii) $\Leftrightarrow$ (iv) $\Leftrightarrow(\mathrm{v})$. By Theorem 3.2 .

(iii) $\Rightarrow$ (ii). If $(X, d)$ is a metric space and $x, y, z, x^{\prime}, y^{\prime}, z^{\prime} \in X$ are such $d\left(x, x^{\prime}\right), d\left(y, y^{\prime}\right), d\left(z, z^{\prime}\right) \leq$ $\frac{1}{2}$, then

$$
\begin{aligned}
\left(x^{\prime} \mid y^{\prime}\right)_{z^{\prime}}= & \frac{1}{2}\left(d\left(z^{\prime}, x^{\prime}\right)+d\left(z^{\prime}, y^{\prime}\right)-d\left(x^{\prime}, y^{\prime}\right)\right) \\
\leq & \frac{1}{2}\left(d\left(z^{\prime}, z\right)+d(z, x)+d\left(x, x^{\prime}\right)+d\left(z^{\prime}, z\right)+d(z, y)+d\left(y, y^{\prime}\right)\right. \\
& \left.\quad+d\left(x, x^{\prime}\right)-d(x, y)+d\left(y^{\prime}, y\right)\right) \\
\leq & \frac{1}{2}(d(z, x)+d(z, y)-d(x, y)+3)=(x \mid y)_{z}+\frac{3}{2} .
\end{aligned}
$$

Let $n \geq 2$ and $x_{0}, \ldots, x_{n}, q \in \bar{\Gamma}_{A} G$. Then there exist $g_{0}, \ldots, g_{n}, p \in G$ such that $d_{A}\left(x_{0}, g_{0}\right), \ldots$, $d_{A}\left(x_{n}, g_{n}\right), d_{A}(q, p) \leq \frac{1}{2}$. By the hypothesis and the inequality proved above, we get

$$
\begin{aligned}
\left(x_{0} \mid x_{n}\right)_{q}^{A} & \geq\left(g_{0} \mid g_{n}\right)_{p}^{A}-\frac{3}{2} \geq \min \left\{\left(g_{0} \mid g_{1}\right)_{p}^{A}, \ldots\left(g_{n-1} \mid g_{n}\right)_{p}^{A}\right\}-\delta^{\prime}-\frac{3}{2} \\
& \geq \min \left\{\left(x_{0} \mid x_{1}\right)_{q}^{A}, \ldots\left(x_{n-1} \mid x_{n}\right)_{q}^{A}\right\}-\delta^{\prime}-3 .
\end{aligned}
$$

Thus we may apply Theorem 3.2 and obtain condition (ii).

We remark that the hyperbolicity condition for a geodesic metric space (all geodesic triangles being $\delta$-thin) is equivalent to all geodesic $m$-gons being $(m-2) \delta$-thin when we fix $m \geq 4$ : for the direct implication we triangulate the polygon, and the converse follows from adding trivial geodesics to a triangle. Thus what distinguishes virtually free groups among arbitrary hyperbolic groups is the capacity of using the same $\delta$ for all polygons simultaneously.

With respect to bigons, we must recall a remarkable theorem of Papasoglu [15, Theorem 1.4], which proved that a finitely generated group $G=\langle A\rangle$ is hyperbolic if and only if there exists some $\delta \geq 0$ such that all geodesic bigons in $\bar{\Gamma}_{A}(G)$ are $\delta$-thin.

As a matter of fact, Papasoglu uses in his statement a synchronous version of thinness (the fellow travel property). We say that two coterminal geodesics $\xi, \xi^{\prime}:[0, s] \rightarrow \bar{\Gamma}_{A}(G)$ fellow travel with bound $\delta$ if $d_{A}\left(t \xi, t \xi^{\prime}\right) \leq \delta$ for every $t \in[0, s]$. Clearly, this implies $\operatorname{Im}(\xi) \subseteq D_{\delta}\left(\operatorname{Im}\left(\xi^{\prime}\right)\right)$.

On the other hand, suppose that $\operatorname{Im}(\xi) \subseteq D_{\varepsilon}\left(\operatorname{Im}\left(\xi^{\prime}\right)\right)$ and let $t \in[0, s]$. Then $d_{A}\left(t \xi, t^{\prime} \xi^{\prime}\right) \leq \varepsilon$ for some $t^{\prime} \in[0, s]$. It is easy to see that $t^{\prime}>t+\varepsilon$ contradicts $\xi^{\prime}$ being a geodesic (we may use $\xi$ and a 
geodesic from $t \xi$ to $t^{\prime} \xi$ to produce a shorter alternative to $\xi^{\prime}$ ), and by symmetry we get $\left|t^{\prime}-t\right| \leq \varepsilon$. Hence

$$
d_{A}\left(t \xi, t \xi^{\prime}\right) \leq d_{A}\left(t \xi, t^{\prime} \xi^{\prime}\right)+d_{A}\left(t^{\prime} \xi^{\prime}, t \xi^{\prime}\right) \leq \varepsilon+\left|t^{\prime}-t\right| \leq 2 \varepsilon
$$

and so the two geodesics fellow travel with bound $2 \varepsilon$. This confirms that the two notions of thinness are indeed equivalent.

However, we must note that Papasoglu's theorem does not hold for arbitrary geodesic metric spaces. Indeed, geodesic bigons are 0-thin in any space where there exists a unique geodesic connecting a given pair of points. This is for instance the case of the Euclidean plane, as noted in [15]. Another counterexample would be $\bar{\Gamma}_{A}(G)$ when $A=\left\{a_{1}, a_{2}, \ldots\right\}$ and $G$ is the group presented by $\left\langle A \mid a_{n}^{2 n+1}(n \geq 1)\right\rangle$ (the free product of cyclic groups of all odd orders).

We end with an application of Theorem 4.1.

Corollary 4.2 Let $G$ be a finitely generated virtually free group and let $H \leq G$. Then the following conditions are equivalent:

(i) $H$ is quasi-convex;

(ii) $H$ is finitely generated.

Proof. (i) $\Rightarrow$ (ii). Since every quasi-convex subgroup of a hyperbolic group is finitely generated [3, Lemma III.Г.3.5].

(ii) $\Rightarrow$ (i). Assume that $G=\langle A\rangle$ and $H=\langle B\rangle$ with $A, B$ finite. Let

$$
M=\max \left\{d_{A}(1, b) \mid b \in B\right\} .
$$

By Theorem 4.1, there exists some $\varepsilon \geq 0$ such that, for all coterminal geodesic $\xi$ and path $\xi^{\prime}$ in $\left(\bar{\Gamma}_{A}(G), d_{A}\right)$,

$$
\operatorname{Im}(\xi) \subseteq D_{\varepsilon}\left(\operatorname{Im}\left(\xi^{\prime}\right)\right)
$$

Let $h, h^{\prime} \in H$ and let $\eta:[0, s] \rightarrow \bar{\Gamma}_{A}(G)$ be a geodesic with $0 \eta=h$ and $s \eta=h^{\prime}$. Write $h^{\prime}=h b_{1} \ldots b_{n}$ for some $b_{1}, \ldots, b_{n} \in \widetilde{B}$. Let $\eta^{\prime}$ be a path in $\bar{\Gamma}_{A}(G)$ concatenating geodesics with endpoints $h b_{1} \ldots b_{i-1}$ and $h b_{1} \ldots b_{i}$ for $i=1, \ldots, n$. Then $\operatorname{Im}(\eta) \subseteq D_{\varepsilon}\left(\operatorname{Im}\left(\eta^{\prime}\right)\right)$. It is immediate that $\operatorname{Im}\left(\eta^{\prime}\right) \subseteq D_{M}(H)$, hence

$$
\operatorname{Im}(\eta) \subseteq D_{\varepsilon+M}(H)
$$

and $H$ is $(\varepsilon+M)$-quasi-convex.

\section{Acknowledgements}

The first author is partially supported by CNPq, PRONEX-Dyn.Syst. and FAPESB (Brazil).

The second author acknowledges support from:

- $\mathrm{CNPq}$ (Brazil) through a BJT-A grant (process 313768/2013-7);

- the European Regional Development Fund through the programme COMPETE and the Portuguese Government through FCT (Fundação para a Ciência e a Tecnologia) under the project PEst-C/MAT/UI0144/2011. 


\section{References}

[1] Y. Antolin, On Cayley graphs of virtually free groups, Groups Complexity Cryptology 3 (2011), $301-327$.

[2] M. Bonk and B. Kleiner, Quasi-hyperbolic planes in hyperbolic groups, Proc. Amer. Math. Soc. 133 (2005), 2491-2494.

[3] M. Bridson and A. Haefliger, Metric Spaces of Non-Positive Curvature, Grundlehren Math. Wissenschaften, Volume 319, Springer, New York, 1999.

[4] V. Diekert and A. Weiss, Context-Free Groups and Bass-Serre Theory, arXiv:1307.8297, to appear in a volume of the series Advanced Courses in Mathematics CRM Barcelona, Birkhäuser.

[5] V. Diekert and A. Weiss, Context-free groups and their structure trees, Internat. J. Algebra Comput. 23 (2013), 611-642.

[6] M. J. Dunwoody, The accessibility of finitely presented groups, Invent. Math. 81(3) (1985), 449-457.

[7] E. Ghys and P. de la Harpe (eds), Sur les Groupes Hyperboliques d'après Mikhael Gromov, Birkhauser, Boston, 1990.

[8] R. H. Gilman, S. Hermiller, D. F. Holt and S. Rees, A characterization of virtually free groups, Arch. Math. 89 (2007), 289-295.

[9] M. J. Greenberg, Euclidean and Non-Euclidean Geometries, 3rd ed., W. H. Freeman and Co., New York, 1994.

[10] M. L. Gromov, Hyperbolic groups, Essays in group theory, Math. Sci. Res. Inst. Publ., vol. 8, Springer, New York, 1987, pp. 75-263.

[11] A. Karrass, A. Pietrowski and D. Solitar, Finitely generated groups with a free subgroup of finite index, J. Austral. Math. Soc. 16 (1973), 458-466.

[12] D. Kuske and M. Lohrey, Logical aspects of Cayley graphs: the group case, Ann. Pure Appl. Logic 131(1-3) (2005), 263-286.

[13] D. E. Muller and P. E. Schupp, Groups, the theory of ends, and context-free languages, $J$. Comput. System Sci. 26(3) (1983), 295-310.

[14] D. E. Muller and P. E. Schupp, The theory of ends, pushdown automata, and second-order logic, Theoret. Comput. Sci. 37(1) (1985), 51-75.

[15] P. Papasoglu, Strongly geodesically automatic groups are hyperbolic, Invent. Math. 121 (1995), 323-334.

[16] F. Rimlinger, Pregroups and Bass-Serre theory, Mem. Amer. Math. Soc. 65(361) (1987), viii+73.

[17] P. Scott and T. Wall, Topological methods in group theory, in: Homological Group Theory (eds. T. Wall and D. Johnson), London Math. Soc. Lecture Notes Series 36, 1979, pp. 137-203. 
[18] P. V. Silva, X. Soler-Escrivà and E. Ventura, Finite automata for Schreier graphs of virtually free groups, preprint, CMUP 2012-2, arXiv:1112.5709.

[19] J. Väisälä, Gromov hyperbolic spaces, Expositiones Math. 23(3) (2005), 187-231. 